\title{
Standing-wave interferometer
}

\author{
H. Stiebig ${ }^{\text {a) }}$ \\ Institute of Photovoltaics, Research Center Jülich, D-52425 Jülich, Germany \\ H. Büchner \\ Institute of Measurement and Sensor Technology, Technische Universität Ilmenau, \\ D-98684 Ilmenau, Germany \\ E. Bunte \\ Institute of Photovoltaics, Research Center Jülich, D-52425 Jülich, Germany \\ V. Mandryka \\ Institute of Measurement and Sensor Technology, Technische Universität Ilmenau, \\ D-98684 Ilmenau, Germany \\ D. Knipp \\ International University Bremen, Department of Science and Engineering, 28759 Bremen, Germany \\ G. Jäger \\ Institute of Measurement and Sensor Technology, Technische Universität Ilmenau, \\ D-98684 Ilmenau, Germany
}

(Received 21 March 2003; accepted 7 May 2003)

An interferometric position sensor was developed using the concept of sampling a standing wave. Interference of a standing wave created in front of a plane mirror can be detected by thin, partly transparent sensors based on amorphous silicon. The optical thickness of the absorption layer is thinner than the wavelength $\lambda$ of the incident light. Detection of minima and maxima of the standing wave can be used to determine the relative displacement of the plane mirror and the detector. For determination of bidirectional fringe counting, two detectors with a certain phase shift were introduced into the standing wave. An integrated solution of two stacked n-i-p diodes and a phase shifter will be presented. The operation principle of the device will be demonstrated by measured Lissajous figures. (C) 2003 American Institute of Physics. [DOI: 10.1063/1.1590732]

Interferometric measurements of the length or related physical values are imported in areas where nanoscale resolution is combined with large measuring ranges from a few tenths of a micrometer to meters. Typical applications are in the area of high precision measurements or positioning of objects with a nanometer accuracy. State-of-the-art technology for precision length measurements and position detection is the Michelson interferometer. Its general operating principle is based on the evaluation of an interference pattern caused by two waves propagating in the same direction. The interference pattern is detected by a photodiode. As an alternative to the standard approach, different concepts for interferometers utilizing the interferences of waves propagating in opposite direction were presented. ${ }^{1,2}$ These setups use a partly transparent diode to detect the standing wave. Bidirectional fringe counting can be achieved, if two thin partly transparent and phase shifted photodetectors are implemented in the standing wave. ${ }^{1}$ The arrangement of the detector system within the standing wave can be significantly simplified when the two diodes and the phase shifter are combined in an integrated sensor system. In the following we will present an integrated detector system based on two stacked thin diodes in combination with a phase shifter. The

${ }^{a)}$ Electronic mail: h.stiebig@fz-juelich.de advantage of the concept arises from its simple setup. It allows reduction of cost and space requirement and takes benefit from its easy optical adjustment.

A standing wave is created in front of a plane mirror by an orthogonally incident laser beam which is reflected onto itself. The interference of these two waves can be described by the superposition of two waves propagating in opposite directions. Assuming a plane mirror the spatial distribution of the light intensity $I(x)$ of a standing wave is given by

$$
I(x)=I_{1}+I_{2}-2 \sqrt{I_{1} I_{2}} \cos \left(\frac{4 \pi}{\lambda} n \cdot x\right) .
$$

$I_{1}$ and $I_{2}$ represent the intensity of the light propagation in $x$ and in opposite direction, respectively. $\lambda$ is the wavelength of the incident light, $n$ denotes the refractive index of the medium, and $x$ shows the position on the optical axis.

The standing wave is detected by the integrated detector system which is realized by two stacked n-i-p diodes with transparent contact layers $\mathrm{TCO}_{\mathrm{I}}, \mathrm{TCO}_{\mathrm{II}}$, and $\mathrm{TCO}_{\mathrm{III}}$ deposited on a glass substrate (Fig. 1). The individual diodes detect an intensity profile $I$ according to Eq. (1). Varying the position of the plane mirror results in a phase shift of the standing wave leading to a change in the optical generation profile within the diodes. The relative displacement of the plane mirror can be determined by counting the minima and maxima of the photocurrent. In case of a detector system which does not disturb the standing wave the interference 


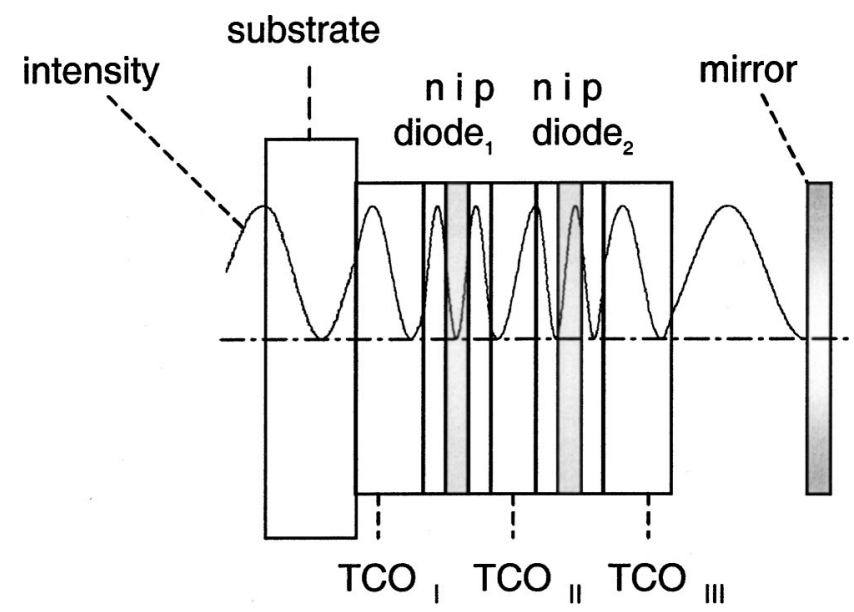

FIG. 1. Sketch of the microinterferometer within a standing wave.

signal detected by each of the diodes should show a sinusoidal dependency on the displacement with a period corresponding to half of the wavelength of the laser beam. This behavior has already been observed by our group for single diodes incorporated into a standing wave. ${ }^{3,4}$ Evaluation of the two phase shifted signals allows to determine the direction of the movement of the mirror. Displaying the photocurrents on the $x$ and $y$ channel of an oscilloscope results in elliptic Lissajous figures. The direction of the travel clockwise or anticlockwise on the screen depends on the direction of the movement of the mirror. If the phase shift of the two detectors and thereby the phase shift of the two signals is attuned to $90^{\circ}$ a Lissajous figure close to a perfect circle is observed. In this ideal case a resolution of the measurement system in the nanometer range can be reached since the two signals form an orthogonal system.

The partly transparent photodiodes of the whole layer stack were prepared of amorphous silicon $(a-\mathrm{Si}: \mathrm{H})$ and its alloy in a n-i-p configuration. The $a-\mathrm{Si}: \mathrm{H}$ based layers were deposited in a multichamber plasma enhanced chemical vapor deposition system at low temperatures $\left(200^{\circ} \mathrm{C}\right)$. The $i$ and $p$-layer were realized of amorphous silicon carbide $(a-\mathrm{SiC}: \mathrm{H})$ by adding methane to the process gas silane. The band gap, $E_{g}$, of the $i$ - and $p$-layer is 2.0 and $1.9 \mathrm{eV}$, respectively. The $n$-layer consists of amorphous silicon $\left(E_{g}=1.75\right.$ $\mathrm{eV}$ ). Doping of the $p$ - and $n$-type layers were achieved by adding trimethylboron and phosphine to the process gases, respectively. ${ }^{5}$ The deposition parameters are described elsewhere. ${ }^{3}$ The first $n-\mathrm{i}-\mathrm{p}$ layer sequence is deposited on a glass substrate coated with smooth $\mathrm{TCO}_{\mathrm{I}}$ (transparent conductive oxide). Afterwards, the second $\mathrm{TCO}_{\mathrm{II}}$ layer was prepared, followed by the second photodiode and then the third contact layer $\mathrm{TCO}_{\mathrm{III}}$. The TCO layers were realized by rfmagnetron sputtered aluminum doped zinc oxide $\mathrm{ZnO}: \mathrm{Al}^{6}$ In order to avoid undesirable absorption losses in the $a-\mathrm{Si}: \mathrm{H}$ system and to achieve a strong modulation of the photocurrent as a consequence of the mirror displacement, ${ }^{3,4}$ (i) the $i$-layer thickness of the diodes must be in the order of $\lambda /(4 n)$ and (ii) the total thickness of the diodes should be around $\lambda /(2 n)$. Since the thickness of the $n-i-p$ diode is below $100 \mathrm{~nm}$, special efforts were attempted to evade shunting problems, e.g., the sputtering rate for the $\mathrm{TCO}_{\mathrm{II}}$ and $\mathrm{TCO}_{\text {III }}$ layers should be low to avoid an ion bombardment of Downloaded 21 Dec 2006 to 134.94.122.39. Redistribution subject the underlying $a$-Si:H layer system. Additionally, $\mathrm{TCO}_{\mathrm{II}}$ and $\mathrm{TCO}_{\text {III }}$ were prepared at room temperature to prevent a deterioration of the optoelectronic properties of the $a-\mathrm{Si}: \mathrm{H}$ layers, which were prepared at $200{ }^{\circ} \mathrm{C}$. The $\mathrm{TCO}_{\mathrm{I}}$ layer was deposited at $400{ }^{\circ} \mathrm{C}$ to achieve a high conductivity of the $\mathrm{ZnO}: \mathrm{Al}$ layer. The layer stacks glass $\mathrm{TCO}_{\mathrm{I}} / \mathrm{n}$-i-p/TCO $/ \mathrm{II} / \mathrm{n}$-i-p/TCO ${ }_{\text {III }}$ (see Fig. 1) were patterned using photolithography and reactive ion etching. The detector system has an active area of around $10 \mathrm{~mm}^{2}$. The photocurrent measurements are performed using a $\mathrm{He}-\mathrm{Ne}$ laser $(\lambda=633 \mathrm{~nm})$ with a power of $1.65 \mathrm{~mW}$.

In the following, the influence of the device design on the properties of the interferometer will be discussed. To avoid disturbance of the standing wave, the layer stack has to be highly transmissive and low reflective for the laser wavelength of $633 \mathrm{~nm}$. These requirements can be fulfilled when the optical thickness of the whole device is $k_{1}^{*} \lambda / 2$ with $k_{1}=1,2,3, \ldots$. Therefore, the optical thickness of the $\mathrm{TCO}_{\mathrm{I}} / \mathrm{n}$-i-p/TCO $/ \mathrm{II}$ - $\mathrm{i}-\mathrm{p} / \mathrm{TCO}_{\text {III }}$ layer stack has to be $\left(k_{2}^{*} \lambda / 4\right) /[d(\mathrm{n}-\mathrm{i}-\mathrm{p})] /\left(k_{3}^{*} \lambda / 2\right) /[d(\mathrm{n}-\mathrm{i}-\mathrm{p})] /\left(k_{2}^{*} \lambda / 4\right)$, with $k_{2}$, $k_{3}=1,2,3, \ldots$. The layers $\mathrm{TCO}_{\mathrm{I}}$ and $\mathrm{TCO}_{\text {III }}$ act on one hand as an antireflection coating and on the other hand as a transparent contact. As a compromise between low reflectivity and high conductivity of the $\mathrm{ZnO}$ layers, $k_{2}$ was chosen to 3 to attain a high cutoff frequency. $\mathrm{TCO}_{\mathrm{II}}$ is embedded between the two diodes and is used as a common contact. In order to optimize the transparency of the system $k_{3}$ was chosen to 1 . Optical calculations show that a transmission of around $80 \%$ can be expected for the layer system. However, deviations in the layer thickness lead to a lower measured transmission at $633 \mathrm{~nm}$ of about $70 \%$.

Beside the optical thickness of the TCO layers, the optical properties of the n-i-p diodes also have an influence on the device performance. The $i$-layer thickness determines (i) the ability to distinguish between the minima and maxima of the standing wave and (ii) the geometric capacitance (necessary for high cutoff frequencies). Therefore, the $i$-layer thickness was chosen to $30 \mathrm{~nm} .^{3,4}$ The measured photocurrent is nearly proportional to the integral of the spatial intensity distribution within the $i$-layer. The $n$-layer thickness of the diodes is $20 \mathrm{~nm}$ and the $p$-layer thickness is $40 \mathrm{~nm}$ to match the total optical thickness of the $a-\mathrm{Si}: \mathrm{H}$ based layer stack to $\lambda / 2$.

Although the total thickness of the n-i-p diode is only around $90 \mathrm{~nm}$ and the $i$-layer is only $30 \mathrm{~nm}$ the dark current density of the individual diodes is very low $\left(I_{\text {dark }}<2\right.$ $\left.\times 10^{-6} \mathrm{~mA} / \mathrm{cm}^{2}\right)$ in the reverse bias range $-0.5 \mathrm{~V}<V<0 \mathrm{~V}$. This value fits well with the dark current of single deposited diodes. Figure 2 shows the quantum efficiency (QE) of both diodes measured from either sides. The QE measured under "direct" illumination conditions (illumination from the side nearest to the diode under test) is higher than the QE at "indirect" illumination conditions (illumination from the side, where the other diode is in front of the diode under test). The lower QE under indirect illumination is due to absorption "losses" in the diode, which is in between the side of incidence of the light and the diode under test. In this case the diode which is arranged in front of the other acts as a transmission filter. Because of the wavelength dependent absorption coefficient, absorption losses are more proto AIP license or copyright, see http://apl.aip.org/apl/copyright.jsp 


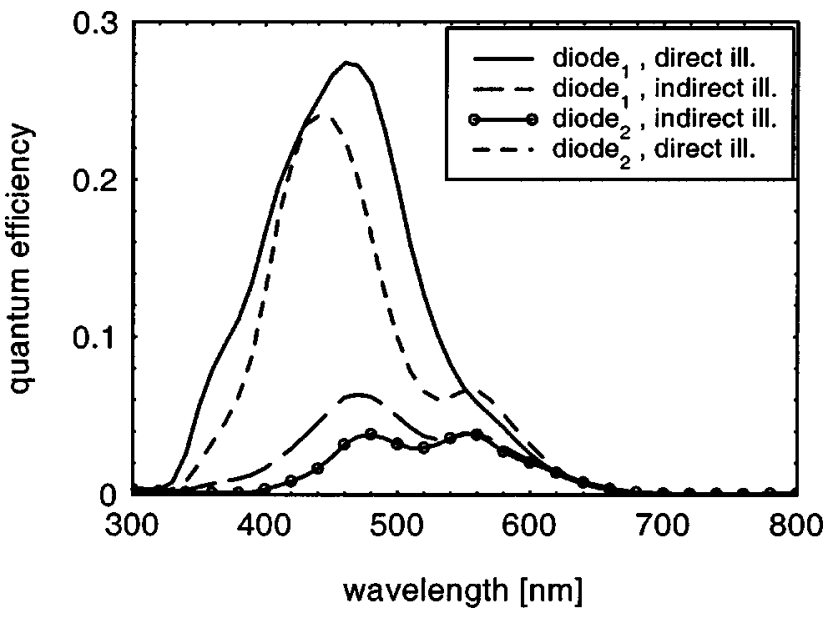

FIG. 2. Measured quantum efficiency of the two n-i-p diodes illuminated from both sides. Direct illumination indicates the measuring results when the light strikes to the side nearest to the diode under test and indirect illumination conditions shows the data, if the light is transmitted by the other diode which is positioned in front of the diode under test.

nounced at shorter wavelengths. For $\lambda>600 \mathrm{~nm}$ similar QEs are measured, which are nearly independent on the direction of illumination. The QE at $633 \mathrm{~nm}$ is about $1 \%-2 \%$.

Next, the detector system is characterized when it is implemented in the standing wave and the plane mirror is moved. An $x y$ plot of the photocurrent of the two diodes is given in Fig. 3. The signals of both diodes vary from $5 \cdots 20-25 \mu \mathrm{A}$, which is within the expected range when using a laser power of $1.65 \mathrm{~mW}$. The relative phase shift of the two currents is about $35^{\circ}$ caused by the different spatial arrangement of the centers of the $i$-layer within the standing wave. It is determined by the optical thickness of half of the $i$-layer of diode $1, p$-layer of diode $1, \mathrm{TCO}_{\mathrm{II}}$ layer, $n$-layer of diode $_{2}$, and half of the $i$-layer of diode $_{2}$. The shift of the center of the ellipse out from the zero point of the coordination system can be attributed to the direct component (dc) of the photocurrent generated within the detectors. The dc part originates from term one and two of Eq. (1) and the finite

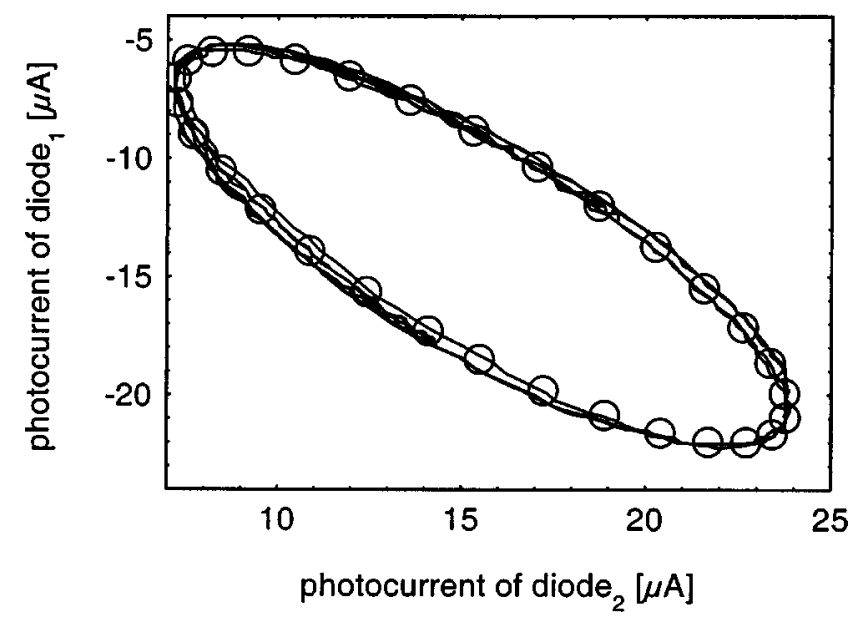

FIG. 3. Measured (lines) and calculated (symbols) elliptic Lissajous figure. $i$-layer thickness. The sign of measured current is a result of the measurement setup. The $\mathrm{TCO}_{\text {II }}$ contact is used as a common ground.

For high transmission the optical thickness of both n-i-p diodes and of the $\mathrm{TCO}_{\mathrm{II}}$ layer should be $\lambda / 2$. However, in this conditions the phase shift between the two photocurrents would be $360^{\circ}$ and the shape of the ellipse shrinks to a single line. The direction of the displacement of the mirror cannot be determined. Thus, we have increased the thickness of the $p$-layer of diode $_{1}$ to around $50 \mathrm{~nm}$ to achieve a phasing between the two photocurrents different from $360^{\circ}$. The $p$-layer thickness was modified because the band gap of the $p$-layer is higher than the $n$-layer. This approach leads to lower absorption losses within the doped layers. The increase in the $p$-layer thickness of diode ${ }_{1}$ by around $10 \mathrm{~nm}$ is reflected in the measured phase shift (Fig. 3). However, a specific optimization of the layer stack is critical because the variations of the deposition parameters lead to thickness variations of the $a-\mathrm{Si}: \mathrm{H}$ based layers, which are in the same order. The shape of the ellipse is in good agreement with the calculated ellipse (symbols) using two sine functions with the same amplitude and a phase shift of $35^{\circ}$. This result indicates that the measured current of the two diodes is also nearly sinusoidal and that the introduction of the sensor does not significantly disturb the interference of the two waves. To obtain a resolution of the interferometer below $\lambda / 2$ the thicknesses of the two $i$-layers, the $p$-layer of diode $_{1}$ and $n$-layer of diode $_{2}$ have to be adapted to obtain phase shifted photocurrents of $90^{\circ}$. This subject is a challenge for the forthcoming works and needs a compromise between optimized optical adjustment of the whole layer stack and the phase shift between the two photocurrents.

In summary, an interferometer based on a stack of two very thin n-i-p diodes with a 30-nm-thick $i$-layer of $a-\mathrm{SiC}: \mathrm{H}$ was realized. The $x y$ display of the two signals form an elliptic Lissajous figure with a corresponding relative phase shift of the centers of the two $i$-layers of around $35^{\circ}$. This result shows that the standing wave generated by two waves propagating in opposite direction can be detected by our developed integrated sensor system and that the photocurrent of both diodes has nearly a sinusoidal shape.

The authors would like to thank O. Kluth, M. Krause, G. Schöpe, and B. Rech for many helpful discussions. The authors gratefully acknowledge the financial support from the "Deutsche Forschungsgemeinschaft" (JA 779/4, Sti 181/ $1-1)$.

${ }^{1}$ H. Büchner, Deutsches Patent DE 3300369 (1983).

${ }^{2}$ M. Sasaki, X. Mi, and K. Hane, Appl. Phys. Lett. 75, 2008 (1999).

${ }^{3}$ H. Stiebig, H.-J. Büchner, E. Bunte, V. Mandryka, D. Knipp, and G. Jäger, Thin Solid Films 427, 152 (2003).

${ }^{4}$ H.-J. Büchner, H. Stiebig, V. Mandryka, E. Bunte, and G. Jäger, Meas. Sci. Technol. 14, 311 (2003).

${ }^{5}$ W. Luft and Y. Tuso, Hydrogenated Amorphous Silicon Alloy Deposition Processes (Marcel Dekker, New York, 1993).

${ }^{6}$ O. Kluth, A. Löffl. S. Wieder, C. Beneking, L. Houben, B. Rech, H. Wagner, S. Waser, J. A. Selvan, and H. Keppner, Proc. 26th IEEE PVSEC (1997) pp. 715-718. 